Relations industrielles

Industrial Relations

\title{
Action Research for Management, by William Foote Whyte and Edith L. Hamilton, Richard D. Irwin, Inc., Homewood, Illinois, 1964, 282 pages.
}

\section{Ronald Pleau}

Volume 20, numéro 4, 1965

URI : https://id.erudit.org/iderudit/027635ar

DOI : https://doi.org/10.7202/027635ar

Aller au sommaire du numéro

Éditeur(s)

Département des relations industrielles de l'Université Laval

ISSN

0034-379X (imprimé)

1703-8138 (numérique)

Découvrir la revue

Citer ce compte rendu

Pleau, R. (1965). Compte rendu de [Action Research for Management, by William Foote Whyte and Edith L. Hamilton, Richard D. Irwin, Inc., Homewood, Illinois, 1964, 282 pages.] Relations industrielles / Industrial Relations, 20(4),

721-722. https://doi.org/10.7202/027635ar

Tous droits réservés @ Département des relations industrielles de l'Université Laval, 1965
Ce document est protégé par la loi sur le droit d'auteur. L'utilisation des services d'Érudit (y compris la reproduction) est assujettie à sa politique d'utilisation que vous pouvez consulter en ligne.

https://apropos.erudit.org/fr/usagers/politique-dutilisation/ 
existe entre le degré de structuration de l'entreprise et le degré de liberté dont jouissent ses membres. Enfin, il s'intéresse à I'organisation en tant que structure contribuant d̀ la promotion, ou développement de l'individu et à sa contribution oux objectifs de la société $\gg$.

Bref, on peut conclure avec le professeur Sayles qu'on ne peut accepter l'affirmation de ceux qui prétendent que la vie dans les grandes organisations conduit à un conformisme excessif et à la dépendance.

Dans cet ouvrage concis et très bien structuré, tout est remis en question concernant les notions traditionnelles de a staff $»$ et a line $»$. On en arrive a la conclusion que si l'on s'y arrête et qu'on réfléchit le moindrement dे ce qui se passe dans les grandes organisations, on ne peut plus maintenant diviser les services de ces dernières en deux types bien délimités.

Une phrase du compositeur mentionne d'ailleurs que: «es conclusions réunies dans cet ouvrage, affirment que la grande organisation industrielle reconcilie l'initiative individuelle avec les nécessités de l'organisation, en donne une image très différente de celle habituellement présentée ou public .

\section{Ronald Pleau}

\section{Profit Sharing in Perspective, B.L. Metzger}

Profit Sharing Research Foundation, 1718

Sherman Avenue, Evanston, Illinois, 1964, 158 poges.

Cette étude sur la distribution des profits dans les petites et moyennes entreprises aux Etots-Unis est le résultat de deux ans de recherche sur les différentes pratiques qui y sont établies. Elle consiste en une enquête qui fut menée dans le but de déterminer l'étendue et l'incidence des programmes de distribution des profits. Cette enquête fut ensuite suivie d'une étude des caractéristiques, des objectifs, des résultats et déficiences des différents plans dont l'acceptation est de plus en plus accentuée.

Quelques-unes de ces découvertes sont assez surprenantes. Ainsi, par exemple, une compagnie sur cinq de celles ayant 50 employés ou plus possède son plan de partoge des profits, $70 \%$ de ces plans sont des programmes couvrant la majorité des employés réguliers et plus de 8 plans sur dix ont été évalués comme étant des succès.

On porte une attention particulière dans différents chapitres à d'autres découvertes de cette enquête, d̀ l'attitude changeante de ceux qui formulent la politique des syndicats et à l'étonnante accélération de la croissance des systèmes de partage des profits.

Comme le mentionne l'auteur, la distribution ou le partage des profits a été défini comme étant une méthode d'augmenter le rendement et diminuer les coûts par lo coopération humaine qui s'opère par la participation directe des employés aux résultats totaux de l'entreprise mesurés par les profits. II existe donc trois types principaux de plans concernant ce sujet lesquels sont décrits dans un premier chapitre.

Puis dans les suivants, c'est la description de la procédure suivie pour l'enquête préliminaire de même qu'une étude des types de programmes de partoge des profits que I'on rencontre le plus souvent dans l'entreprise américaine, de ses caractéristiques générales et de la participation des employés à ces plans.

Cette première partie est couronnée par un échantillon des plans i.e. un genre de tableau sommaire des différents plans étudiés dans les chapitres précédents.

Dans ce qu'on pourrait appelée une seconde partie, on analyse les résultats de cette enquête et ce sont les objectifs du plan, l'évaluation qu'en fait le management, les effets des programmes, l'attitude du syndicat vis-à-vis le partage des profits et enfin les conditions de succès de ces plans et programmes qui sont l'objet d'un examen plus approfondi. Un dernier chapitre vient compléter cette analyse avec une brève étude des autres genres de programmes de bénéfices.

De courts résumés à la fin de chaque chapitre sont présentés dans le but de fournir au lecteur une image globale ou permettre une révision rapide des buts du chapitre bien qu'ils soient loin de constituer des substituts à l'information beaucoup plus détaillée contenue dans ces derniers.

\section{Ronald Pleau}

Action Research for Management, by William Foote Whyte and Edith $L$. Hamilton, Richard D. Irwin, Inc., Homewood, Illinois, 1964, 282 pages.

Publié en fonction des gens intéressés aux relations humaines dans l'industrie, à la sociologie industrielle et à l'administration du personnel, ce volume est un compte-rendu d'un projet de recherche dan!: l'industrie des 
services d'hôtellerie qui peut contribuer au processus de transformation de la théorie de l'organisation selon l'opinion des auteurs cux-mêmes.

Le projet prit naissonce lorsque le viceprésident d'un hôtel américain demanda ò l'un des auteurs de bâtir un plan de recherche concernant les relations humaines dans son entreprise. II était fasciné par l'idée que les relations entre les gens pouvaient être sujettes à une étude. Au début, donc, les auteurs, qui incidemment ont trovaillé ensemble dans cet hôtel pour y étudier les relations inter-personnelles, ne savaient pas quel point ils allaient développer de façon particulière. Aussi le premier chapitre de ce volume est-il consacré à la narration des événements qui ont conduit à l'élaborotion de leur plan de recherche sur le "management » et de la façon dont o débuté leurs trovaux.

Comme dans toute analyse d'un problème, on présente ensuite les caractéristiques de l'entreprise en question et les acteurs y évoluant, en essayant de découvrir où se trouvaient alors les difficultés qui pouvaient être solutionnées.

La première étape à entreprendre pour ces chercheurs consistait donc à clarifier leur rôle, à réorganiser les activités du personnel, à établir leurs relations avec la haute direction et à s'occuper des griefs afin de mettre sur pied leur projet.

Le second développement de cet ouvrage est consacré à la description du travail et à la présentation des résultats de la recherche à mesure qu'elle évoluait dans les différents départements de l'entreprise en question à savoir: le restaurant, la cuisine, le bureau de réception et le bar. De plus, dans I'un des chapitres constituant cette partie, les tâches, les activités, les attitudes, les relations de travail des caissiers et des vérificateurs sont examinées avec soin.

Dans une dernière section on retrouve enfin une évaluation complète de tout ce qui s'est fait, de la stratégie employée, des résultats obtenus et des difficultés rencontrées, pour couronner le tout par un essai d'intégration de cette recherche dans les processus de leodership et de groupe, d'application de la théorie à l'action et de généralisation de cette approche particulière.

Bref, on peut dire que cette étude peut être de lecture intéressante mais ne constitue qu'une approche unique et très personnelle au problème des relations humaines dont on ne pourrait tirer aucun modele d'opplicotion générale.

\section{Ronald Pleau}

\section{Procedures and Policies of the New York State Labor Relations Board, Kurt L. Hanslowe, Cornell University, Ithaca, New York, 1964, 216 pages.}

Ce volume dont l'un des buts est de fournir un guide concernant les procédures des commissions de relations de travail, fut préparé à cause de l'importance toujours grandissante que prend la commission des relations de travail de l'étct de New-York vis-ò-vis les employeurs, les employés et les syndicats et à cause de la rareté des écrits existants en motière de tribunaux administratifs.

Globalement le contenu de cet ouvrage est constitué d'une étude des problèmes d'administration publique et de la loi administrative. On se limite cependant ici au cas de l'étot de New-York dans le champ spécifique des relations patronales-ouvrières.

Comme il se devait, l'auteur nous fait d'abord connaître tous les détails de la loi des relations ouvrières de cet état en attachant une importance toute particulière ò la section 704 de cette dernière qui concerne les protiques de travail interdites. Pour ajouter à la valeur de ce chapitre, il procède ensuite à quelques comparaisons entre cette loi et celle existant au niveau national en s'arrêtant plus longuement sur l'analyse du problème posé par les syndicats de boutique.

C'est à la description de l'organisation et du personnel de la commission des relations de travail qu'est consacré le deuxièrne chapitre dans lequel sont traités des sujets aussi variés mais aussi logiquement reliés que les suivants: la place de la commission dans le département (ou ministère) du travail, la commission des relations de travail et la commission de conciliation, les membres de la commission, et le personnel subordonné.

Ainsi oprès nous avoir présenté les acteurs et leurs instruments de travail, nous passons ò l'examen de leur rôle lorsque l'auteur s'attarde à nous expliquer la procédure antérieur à l'audition formelle pour le cas des pratiques interdites de travail. II soulève ici les problèmes relatifs ou chef d'accusation et à l'enquête informelle menée en ropport avec ce dernier.

Toujours en rapport ovec le même genre de cas, ce sont ensuite les informations concernant l'audition comme telle, la procédure 